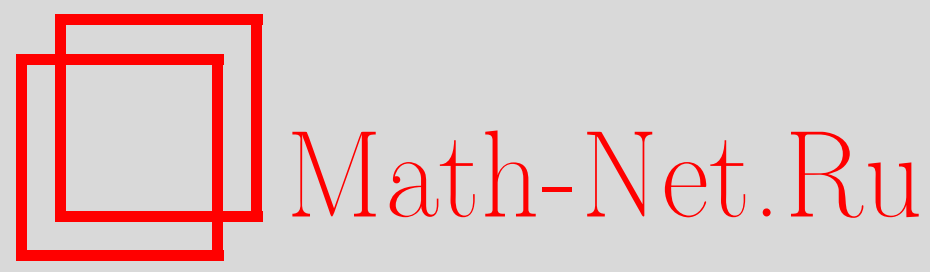

С. А. Дроздовский, Топологическая структура на множестве непрерывных функций с различными областями определения, Матем. заметки, 1999, том 66, выпуск 1, 76-88

DOI: https://doi.org/10.4213/mzm1143

Использование Общероссийского математического портала Math-Net.Ru подразумевает, что вы прочитали и согласны с пользовательским соглашением http://www.mathnet.ru/rus/agreement

Параметры загрузки:

IP: 107.22 .136 .117

26 апреля 2023 г., 11:00:02

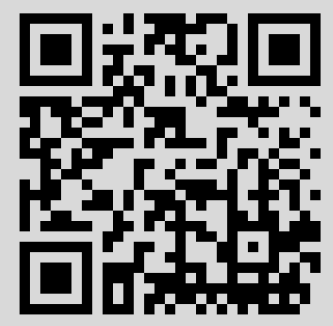




\section{ТОПОЛОГИЧЕСКАЯ СТРУКТУРА НА \\ МНОЖЕСТВЕ НЕПРЕРЫВНЫХ ФУНКЦИЙ С \\ РАЗЛИЧНЫМИ ОБЛАСТЯМИ ОПРЕДЕЛЕНИЯ}

\section{С. А. Дроздовский}

Построено топологическое пространство непрерывных функций, являющееся обобщением ранее изученного пространства функций, определенных на замкнутых отрезках. Изучены свойства типа метризуемости этого пространства. Полученные результаты применимы в топологической теории обыкновенных дифференциальных уравнений.

Библиограффия: 5 названий.

Изученное в данной работе новое топологическое пространство функций $F(U)$ является обобщением известных пространств $C_{s}(U), C_{s}^{+}(U), C_{s}^{-}(U), C_{s}^{ \pm}(U)$ (см. определения ниже), которые являются базовыми в топологической теории обыкновенных дифференциальных уравнений. Пространство $C_{s}(U)$ подробно описано в $[1]$ и $[2], C_{s}^{+}(U)$ и $C_{s}^{-}(U)$ использовались в [3], пространство $C_{s}^{ \pm}(U)$ рассмотрено в [4]. Там же изложен ряд результатов теории, полученных при помощи этих пространств.

Множество $F(U)$ есть объединение множеств $C_{s}(U), C_{s}^{+}(U), C_{s}^{-}(U), C_{s}^{ \pm}(U)$, а введенная на $F(U)$ топология индуцирует на них их собственные топологии. Рассмотренное в конце статьи пространство $F^{*}(U)$ обобщает аналогичную конструкцию, использовавшуюся в [4].

В работе исследованы свойства типа метризуемости пространства $F(U)$ и его подмножеств.

1. Пространство $F(U)$. Пусть $U$ - открытое подмножество произведения $\mathbb{R} \times M$, где $\mathbb{R}$ - множество действительных чисел, а $M$ - метрическое сепарабельное локально-компактное пространство. Мы будем рассматривать непрерывные функции из $\mathbb{R}$ в $M$, определенные на различных сегментах, интервалах и полуинтервалах, графики которых являются подмножествами множества $U$. Обозначим через $C_{s}(U)$ множество всех непрерьвных функций вида $z:\left[t_{1}, t_{2}\right] \rightarrow M$, через $C_{s}^{+}(U)$ и $C_{s}^{-}(U)$ пространства всех непрерьвных функций вида $z:\left[t_{0}, t\right) \rightarrow M$ и $z:\left(t, t_{0}\right] \rightarrow M$ соответственно, графики которых замкнуты в $U ; t$ может принимать бесконечные значения. Символом $C_{s}^{ \pm}(U)$ обозначим множество всех непрерьвных функций $\left(t_{1}, t_{2}\right) \rightarrow M$ с замкнутыми в $U$ графиками. Множество $F(U)$ определим как объединение множеств $C_{s}(U), C_{s}^{+}(U), C_{s}^{-}(U)$, $C_{s}^{ \pm}(U)$.

Топология, которую мы введем на $F(U)$, соответствует сходимости, являющейся естественньм обобщением равномерной сходимости функций, определенных на отрезке. 
Пусть $z \in C_{s}(U)$. Последовательность $\left\{z_{i}: i<\omega\right\} \subset F(U)$ сходится к $z$ тогда и только тогда, когда существует такое $i_{1}$, что для всех $i>i_{1} z_{i} \in C_{s}(U)$, семейство $\left\{z_{i}\right.$ : $\left.i_{1}<i<\omega\right\}$ равностепенно непрерывно, и для каждых замкнутого отрезка $I \subset\langle\pi(z)\rangle$, где $\pi(z)$ - область определения $z$, и открытого интервала $\widehat{I} \supset \pi(z)$ существует такое $i_{2}$, что для всех $i>i_{2} I \subseteq \pi\left(z_{i}\right) \subset \widehat{I}$, и $\left\|\left.z_{i}\right|_{I}-\left.z\right|_{I}\right\| \rightarrow 0$ при $i \rightarrow \infty$, где \|\| обозначает норму равномерной сходимости.

Пусть $z \in C_{s}^{+}(U)$. Последовательность $\left\{z_{i}: i<\omega\right\}$ сходится к $z$ в том и только в том случае, если существует такое $i_{1}$, что для всех $i>i_{1} z_{i} \in C_{s}(U) \cup C_{s}^{+}(U)$, и для каждого замкнутого отрезка $I \subset\langle\pi(z)\rangle$ и открытого интервала $\widehat{I} \supset \pi(z)$ существует такое $i_{2}$, что для всех $i>i_{2} I \subseteq \pi\left(z_{i}\right) \subset \widehat{I}$, семейство $\left\{\left.z_{i}\right|_{\pi\left(z_{i}\right) \cap(-\infty, \sup I]}: i_{2}<i<\omega\right\}$ равностепенно непрерьвно, и $\left\|\left.z_{i}\right|_{I}-\left.z\right|_{I}\right\| \rightarrow 0$ при $i \rightarrow \infty$.

Сходимость к элементам из $C_{s}^{-}(U)$ определяется аналогично. Достаточно в определении для $C_{s}^{+}(U)$ заменить все символы “+" на символ “-”, а также полуинтервал $(-\infty, \sup I]$ на полуинтервал $[\inf I,+\infty)$.

В случае, когда $z \in C_{s}^{ \pm}(U)$, последовательность $\left\{z_{i}: i<\omega\right\}$ элементов из $F(U)$ сходится к $z$ тогда и только тогда, когда для любого замкнутого отрезка $I \subset \pi(z)$ существует такое $i_{1}$, что для всех $i>i_{1} I \subseteq \pi\left(z_{i}\right)$, и $\left\|\left.z_{i}\right|_{I}-\left.z\right|_{I}\right\| \rightarrow 0$.

Опишем теперь базу топологии пространства $F(U)$, соответствующую заданной сходимости.

Сначала определим для каждого $z \in F(U)$ множества $c_{s}(z), c_{s}^{+}(z), c_{s}^{-}(z)$.

Пусть $z \in C_{s}(U), \pi(z)=\left[t_{1}, t_{2}\right]$. Тогда

$$
\begin{gathered}
c_{s}(z)=\left\{\left.z\right|_{\left[t_{1}^{\prime}, t_{2}^{\prime}\right]}: t_{1} \leqslant t_{1}^{\prime} \leqslant t_{2}^{\prime} \leqslant t_{2}\right\}, \quad c_{s}^{+}(z)=\left\{\left.z\right|_{\left[t_{1}, t\right]}: t_{1} \leqslant t \leqslant t_{2}\right\}, \\
c_{s}^{-}(z)=\left\{\left.z\right|_{\left[t, t_{2}\right]}: t_{1} \leqslant t \leqslant t_{2}\right\} .
\end{gathered}
$$

Если $z \in C_{s}^{+}(U), \pi(z)=\left[t_{1}, t_{2}\right)$, то

$c_{s}(z)=\left\{\left.z\right|_{\left[t_{1}^{\prime}, t_{2}^{\prime}\right]}: t_{1} \leqslant t_{1}^{\prime} \leqslant t_{2}^{\prime}<t_{2}\right\}, \quad c_{s}^{+}(z)=\left\{\left.z\right|_{\left[t_{1}, t\right]}: t_{1} \leqslant t<t_{2}\right\}, \quad c_{s}^{-}(z)=\varnothing$.

Если $z \in C_{s}^{-}(U), \pi(z)=\left(t_{1}, t_{2}\right]$, то

$c_{s}(z)=\left\{\left.z\right|_{\left[t_{1}^{\prime}, t_{2}^{\prime}\right]}: t_{1}<t_{1}^{\prime} \leqslant t_{2}^{\prime} \leqslant t_{2}\right\}, \quad c_{s}^{-}(z)=\left\{\left.z\right|_{\left[t_{,} t_{2}\right]}: t_{1}<t \leqslant t_{2}\right\}, \quad c_{s}^{+}(z)=\varnothing$.

Для $z \in C_{s}^{ \pm}(U), \pi(z)=\left(t_{1}, t_{2}\right)$,

$$
c_{s}(z)=\left\{\left.z\right|_{\left[t_{1}^{\prime}, t_{2}^{\prime}\right]}: t_{1}<t_{1}^{\prime} \leqslant t_{2}^{\prime}<t_{2}\right\}, \quad c_{s}^{+}(z)=c_{s}^{-}(z)=\varnothing .
$$

В дальнейшем мы будем обозначать график элемента $z \in F(U)$ и сам элемент одним и тем же символом $z$.

Базу окрестностей точки $z \in C_{s}(U)$, как было определено в [1], составляют множества вида $G(z, V)=V^{0}=C_{s}(U) \cap V$, где $V$ - некоторая окрестность Виеториса точки $z$ в $\exp (U)$.

Пусть $z \in C_{s}^{+}(U)$. Элемент $6 а з ы$ в этой точке определим как множество

$$
G(z, y, V)=V^{+}=\left\{x \in C_{s}(U) \cup C_{s}^{+}(U): c_{s}^{+}(x) \cap V \neq \varnothing\right\},
$$

где $V$ - виеторисовская окрестность какого-либо элемента $y \in c_{s}^{+}(z)$. 
Окрестность точки $z \in C_{s}^{-}(U)$ получается из $G(z, y, V), z \in C_{s}^{+}(U)$, заменой всех символов "+" на "-".

Для $z \in C_{s}^{ \pm}(U)$ окрестность

$$
G(z, y, V)=V^{ \pm}=\left\{x \in F(U): c_{s}(x) \cap V \neq \varnothing\right\},
$$

где $V$ - окрестность Виеториса точки $y \in c_{s}(z)$.

Заметим, что подмножества $C_{s}(U), C_{s}(U) \cup C_{s}^{+}(U), C_{s}(U) \cup C_{s}^{-}(U)$ открыты в $F(U)$.

УТВЕРЖДЕНИЕ 1. Пространство $F(U)$ имеет счетную базу.

ДоКАЗАТЕЛЬСтво. Пространство $\exp (U)$, как известно, обладает счетной базой. Пусть $\mathcal{B}$ - некоторая счетная база пространства $\exp (U)$. Семейство $\left\{V^{0}, V^{+}, V^{-}, V^{ \pm}\right.$: $V \in \mathcal{B}\}$, в чем нетрудно убедиться, является счетной базой для $F(U)$.

2. Обобщенная метризуемость. В дальнейшем нам понадобятся некоторые факты, касающиеся свойств типа метризуемости.

ОПРЕДЕЛЕнИЕ. Топологическое пространство $X$ назьвается обобщенно метризуемым или $о$-метризуемым о-метрикой $\delta$, если существует неотрицательная функция $\delta: X \times X \rightarrow \mathbb{R}^{+}$, обладающая свойствами:

1) $\delta(x, y)=0$ тогда и только тогда, когда $x=y$;

2) подмножество $U$ пространства $X$ открыто тогда и только тогда, когда для любого $x \in U$ существует такое $\varepsilon>0$, что $\{y \in X: \delta(x, y)<\varepsilon\} \subseteq U$.

Если выполняются свойства 1) и 2) и, кроме того, $\delta(x, y)=\delta(y, x)$ для любых $x, y \in X$, то пространство $X$ симметризуемо симметрикой $\delta$.

Если помимо свойств 1) и 2) выполняется свойство: для всех $x, y, z \in X \delta(x, y) \leqslant$ $\delta(x, z)+\delta(z, y)$, то o-метрика $\delta$ назьвается $\Delta$-метрикой, а пространство $X \Delta$-метрuзуемым.

Условие 2) равносильно условию $2^{\prime}$ ). Подмножество $F$ пространства $X$ замкнуто тогда и только тогда, когда для любого $x \notin F \delta(x, F)=\inf \{\delta(x, y): y \in F\}>0$.

Очевидно следующее утверждение.

УТВЕРЖДЕНИЕ 2. о-метризуемое топологическое пространство удовлетворяет аксиоме отделимости $T_{1}$.

ЛЕмма 1. Пусть топологическое пространство $X$ о-метризуемо о-метрикой $\delta$ такой, что функиия $\delta^{\prime}(x, y)=\delta(y, x)$ тоже является о-метрикой. Тогда $X$ симметризуемо.

ДокАЗАтЕльство. Симметрика на $X$ задается очевидным образом формулой $r(x, y)$ $=\max \{\delta(x, y) ; \delta(y, x)\}$.

ЛЕмма 2. Если топологическое пространство Х о-метризуемо о-метрикой $\delta$, причем функиия

$$
r(x, y)=\inf _{\left\{z_{1}, \ldots, z_{n}\right\} \subseteq X, n<\omega}\left\{\delta\left(x, z_{1}\right)+\delta\left(z_{1}, z_{2}\right)+\cdots+\delta\left(z_{n}, y\right)\right\}
$$

является о-метрикой, то $X \quad \Delta$-метризуемо и $r$ является соответствующей $\Delta$-метрикой. 
ДокАЗАТЕЛЬСТво. Имеем

$$
\begin{aligned}
r(x, z)+r(z, y)= & \inf _{\left\{u_{1}, \ldots, u_{n}\right\} \subseteq X, n<\omega}\left\{\delta\left(x, u_{1}\right)+\delta\left(u_{1}, u_{2}\right)+\cdots+\delta\left(u_{n}, z\right)\right\} \\
& +\inf _{\left\{v_{1}, \ldots, v_{m}\right\} \subseteq X, m<\omega}\left\{\delta\left(z, v_{1}\right)+\delta\left(v_{1}, v_{2}\right)+\cdots+\delta\left(v_{m}, y\right)\right\} \\
\geqslant & \inf _{\left\{u_{1}, \ldots, u_{n}, v_{1}, \ldots, v_{m}\right\} \subseteq X, n+m<\omega}\left\{\delta\left(x, u_{1}\right)+\delta\left(u_{1}, u_{2}\right)+\cdots+\delta\left(u_{n}, z\right)\right. \\
& \left.+\delta\left(z, v_{1}\right)+\delta\left(v_{1}, v_{2}\right)+\cdots+\delta\left(v_{m}, y\right)\right\} \\
\geqslant & \inf _{\left\{u_{1}, \ldots, u_{n}, w, v_{1}, \ldots, v_{m}\right\} \subseteq X, n+m<\omega}\left\{\delta\left(x, u_{1}\right)+\delta\left(u_{1}, u_{2}\right)+\cdots+\delta\left(u_{n}, w\right)\right. \\
& \left.+\delta\left(w, v_{1}\right)+\delta\left(v_{1}, v_{2}\right)+\cdots+\delta\left(v_{m}, y\right)\right\}=r(x, y) .
\end{aligned}
$$

СлЕДСТВИЕ. Если в условиях леммы $2 \delta$-симметрика, то пространство $X$ метризуемо.

ЛЕмма 3 [5]. . Пусть топологическое пространство $X$ $\Delta$-метризуемо $\Delta$-метрикой $\delta$. Кроме того, $\delta$ обладает свойством: для любой точки $x \in X$ и произвольной последовательности $\left\{y_{n}: n<\omega\right\} \subseteq X$ из того, что $\lim _{n \rightarrow \infty} \delta\left(x, y_{n}\right)=0$, cледует, что $\lim _{n \rightarrow \infty} \delta\left(y_{n}, x\right)=0$. Тогда $X$ метризуемо.

Доказательство заключается в проверке того, что $r(x, y)=\max \{\delta(x, y) ; \delta(y, x)\}$ есть метрика на $X$.

Лемма 4 [5]. . Свойство топологического пространства "быть о-метризуемым данной о-метрикой $\delta$ "наследственно по замкнутым подмножествам.

ДокАЗАТЕЛЬСТво. Пусть $Y$ - замкнутоеподпространство $o$-метризуемого $o$-метрикой $\delta$ пространства $X$, и пусть $F \subset Y$. Если $F$ замкнуто в $Y$, то оно замкнуто и в $X$. Следовательно, для любой точки $x \in Y \backslash F \subseteq X \backslash F \delta(x, F)>0$. Если $F$ не замкнуто в $Y$, то оно не замкнуто и в $X$, т.е. существует точка $x \in X \backslash F$ такая, что $\delta(x, F)=0$ и, следовательно, $\delta(x, Y)=0$. Но $Y$ замкнуто, поэтому $x \in Y$.

Более подробная информация об о-метризуемости содержится в [5].

3. Свойства типа метризуемости пространства $F(U)$. Здесь мы сконструируем ряд $O$-метрик для различных подмножеств пространства $F(U)$.

Tеорема 1. Пространства $F^{+}(U)=C_{s}(U) \cup C_{s}^{+}(U)$ u $F^{-}(U)=C_{s}(U) \cup C_{s}^{-}(U)$ метризуемы.

ДокАзАТЕЛьство. Зададим метрику на $F^{+}(U)$ явным образом. Для $F^{-}(U)$ метрика строится аналогично.

Поскольку пространство $M$ локально-компактно, существует одноточечная компактификация Александрова $U^{*}=U \cup\left\{u_{\infty}\right\}$ множества $U$, которая, так же как и $M$, имеет счетную базу и потому метризуема некоторой метрикой $\rho$. Соответственно пространство $\exp _{c} U^{*}$ метризуемо метрикой $H$.

Определим модифицированную метрику Хаусдорфа $h$ в пространстве $C_{s}(U)$ :

$$
\begin{gathered}
h(x, y)=\max \{g(x, y) ; g(y, x)\}, \\
g(x, y)=\max \left\{\sup _{v \in c_{s}(x)} \inf _{s \in c_{s}(y)} H(v, s) ; \sup _{v \in c_{s}^{+}(x)} \inf _{s \in c_{s}^{+}(y)} H(v, s) ; \sup _{v \in c_{s}^{-}(x)} \inf _{s \in c_{s}^{-}(y)} H(v, s)\right\} .
\end{gathered}
$$


Нетрудно проверить, что $h$ - метрика, задающая топологию Виеториса на $C_{s}(U)$.

Докажем теорему для $F^{+}(U)$. Для $F^{-}(U)$ доказательство аналогично.

Введем в пространстве $F^{+}(U)$ симметрику, определенную формулой

$$
\delta(x, y)=\inf _{(v, s) \in D\left(f_{x y}\right)} f_{x y}(v, s),
$$

где $D\left(f_{x y}\right) \subseteq c_{s}(x) \times c_{s}(y)$ - область определения функции $f_{x y}$.

Для $x, y \in C_{s}(U)$

$$
D\left(f_{x y}\right)=\{x\} \times\{y\}, \quad f_{x y}(v, s)=f_{y x}(s, v)=h(v, s) .
$$

В случае, когда $x \in C_{s}(U), y \in C_{s}^{+}(U)$ или $x, y \in C_{s}^{+}(U)$,

$$
\begin{aligned}
D\left(f_{x y}\right) & =c_{s}^{+}(x) \times c_{s}^{+}(y), \quad D\left(f_{y x}\right)=c_{s}^{+}(y) \times c_{s}^{+}(x), \\
f_{x y}(v, s) & =f_{y x}(s, v)=h(v, s)+\rho\left(v(\sup \pi(v)), u_{\infty}\right)+\rho\left(s(\sup \pi(s)), u_{\infty}\right) .
\end{aligned}
$$

Замкнутость графика элемента $z \in F^{+}(U)$ в $U$ означает, что либо $z \in C_{s}(U)$ и $\rho\left(z, u_{\infty}\right)>0$, либо $z \in C_{s}^{+}(U)$, и при $t \rightarrow \sup \pi(z) \rho\left(z(t), u_{\infty}\right) \rightarrow 0$. Отсюда с очевидностью следует, что $\delta$ соответствует заданной топологии.

Докажем, что $о$-метрика $\delta$ удовлетворяет условию леммы 2 .

Пусть $x$ и $y$-какие-либо два элемента множества $F^{+}(U)$ и $\left\{z_{1}, z_{2}, \ldots, z_{n}\right\} \subseteq F^{+}(U)$ - конечное упорядоченное множество такое, что $z_{1}=x, z_{n}=y, n>2$. Для каждой пары соседних элементов $z_{i}, z_{i+1}, 1 \leqslant i \leqslant n-1$, выберем в соответствии с определением величины $\delta$ пару компактов $\left(v_{i(i+1)}, v_{(i+1) i}\right) \in D\left(f_{z_{i} z_{i+1}}\right)$, удовлетворяющих неравенству

$$
\left|f_{z_{i} z_{i+1}}\left(v_{i(i+1)}, v_{(i+1) i}\right)-\delta\left(z_{i}, z_{i+1}\right)\right|<\frac{1}{2} \delta\left(z_{i}, z_{i+1}\right) .
$$

Рассмотрим два возможных варианта.

1) $x, y \in C_{s}(U)$. Пусть $i_{0}$ - такой наименьший номер, что для всех $i, i_{0} \leqslant i \leqslant n$, $z_{i} \in C_{s}(U)$.

Если $i_{0}=1$, то

$$
\sum_{i=1}^{n-1} \delta\left(z_{i}, z_{i+1}\right)=\sum_{i=1}^{n-1} h\left(z_{i}, z_{i+1}\right) \geqslant h\left(z_{1}, z_{n}\right)=\delta(x, y)
$$

Пусть $i_{0}>1$; тог да $z_{i_{0}-1} \in C_{s}^{+}(U)$.

Если $h\left(z_{i_{0}}, y\right)<\frac{1}{2} \rho\left(y, u_{\infty}\right)$, то, учитьвая определение величины $h$, имеем

$$
\begin{aligned}
\rho\left(v_{i_{0}\left(i_{0}-1\right)}\left(\sup \pi\left(v_{i_{0}\left(i_{0}-1\right)}\right)\right), u_{\infty}\right) & \geqslant \rho\left(y, u_{\infty}\right)-\rho\left(v_{i_{0}\left(i_{0}-1\right)}\left(\sup \pi\left(v_{i_{0}\left(i_{0}-1\right)}\right)\right), y\right) \\
& \geqslant \rho\left(y, u_{\infty}\right)-h\left(z_{i_{0}}, y\right)>\frac{1}{2} \rho\left(y, u_{\infty}\right) .
\end{aligned}
$$

Из неравенства $(3.1) \delta\left(z_{i}, z_{i+1}\right)>\frac{2}{3} \rho\left(v_{(i+1) i}\left(\sup \pi\left(v_{(i+1) i}\right)\right), u_{\infty}\right)$ для каждого $i<n$. Следовательно,

$$
\sum_{i=1}^{n-1} \delta\left(z_{i}, z_{i+1}\right)>\delta\left(z_{i_{0}-1}, z_{i_{0}}\right)>\frac{1}{3} \rho\left(y, u_{\infty}\right)
$$


Если $h\left(z_{i_{0}}, y\right) \geqslant \frac{1}{2} \rho\left(y, u_{\infty}\right)$, то $i_{0}<n$ и

$$
\sum_{i=1}^{n-1} \delta\left(z_{i}, z_{i+1}\right) \geqslant \sum_{i=i_{0}}^{n-1} \delta\left(z_{i}, z_{i+1}\right)=\sum_{i=i_{0}}^{n-1} h\left(z_{i}, z_{i+1}\right) \geqslant h\left(z_{i_{0}}, z_{n}\right) \geqslant \frac{1}{2} \rho\left(y, u_{\infty}\right)
$$

2 ) Хотя бы один из элементов $x, y$ принадлежит $C_{s}^{+}(U)$. Используя определение метрики $h$ и неравенство $(3.1)$, легко можно проверить, что среди компактов $v_{i(i+1)}, v_{i(i-1)}$ найдется такой компакт $w_{i_{0}}$, для которого можно последовательно построить содержащую его цепь $\left\{w_{i} \in c_{s}\left(z_{i}\right): 1 \leqslant i \leqslant n\right\}$, для которой при каждом $i, 1<i<n$,

$$
w_{i} \subseteq v_{i(i-1)} \cap v_{i(i+1)}, \quad w_{1} \subseteq v_{12}, \quad w_{n} \subseteq v_{n(n-1)}
$$

и $h\left(w_{i}, w_{i+1}\right)<\frac{3}{2} \delta\left(z_{i}, z_{i+1}\right)$ при $1 \leqslant i \leqslant n-1$. Из неравенства треугольника имеем

$$
\begin{aligned}
h\left(w_{1}, w_{n}\right) & \leqslant \sum_{i=1}^{n-1} h\left(w_{i}, w_{i+1}\right), \\
\rho\left(w_{1}\left(\sup \pi\left(w_{1}\right)\right), u_{\infty}\right) & <\sum_{i=1}^{n-1} h\left(w_{i}, w_{i+1}\right)+\rho\left(w_{i_{0}}\left(\sup \pi\left(w_{i_{0}}\right)\right), u_{\infty}\right), \\
\rho\left(w_{n}\left(\sup \pi\left(w_{n}\right)\right), u_{\infty}\right) & <\sum_{i=1}^{n-1} h\left(w_{i}, w_{i+1}\right)+\rho\left(w_{i_{0}}\left(\sup \pi\left(w_{i_{0}}\right)\right), u_{\infty}\right) .
\end{aligned}
$$

Следовательно, учитывая, что $h\left(w_{i}, w_{i+1}\right)<\frac{3}{2} \delta\left(z_{i}, z_{i+1}\right)$ и $\rho\left(w_{i_{0}}\left(\sup \pi\left(w_{i_{0}}\right)\right), u_{\infty}\right)<$ $\frac{3}{2} \delta\left(z_{i_{0}}, z_{i_{0} \pm 1}\right)$ из $(3.1)$, получим

$$
\begin{aligned}
\delta(x, y)= & \delta\left(z_{1}, z_{n}\right)<\rho\left(w_{1}\left(\sup \pi\left(w_{1}\right)\right), u_{\infty}\right) \\
& +\rho\left(w_{n}\left(\sup \pi\left(w_{n}\right)\right), u_{\infty}\right)+h\left(w_{1}, w_{n}\right)<7 \frac{1}{2} \sum_{i=1}^{n-1} \delta\left(z_{i}, z_{i+1}\right) .
\end{aligned}
$$

Таким образом, мы доказали, что для любого фиксированного $x \in F^{+}(U)$ и переменного $y \in F^{+}(U)$ из $r(x, y) \rightarrow 0$ следует, что $\delta(x, y) \rightarrow 0$, где $r(x, y)$ - величина, определенная в условии леммы 2 . Обратная импликация очевидна. Поэтому $r$ является $O$-метрикой, и симметрика $\delta$ удовлетворяет условиям следствия из леммы 2 , откуда $F^{+}(U)$ метризуемо метрикой $r$.

\section{Теорема 2. Пространство $F(U) \Delta$-метризуемо.}

ДокАЗАТЕЛЬСтво. Чтобы доказать утверждение, построим соответствующую $\Delta$-метрику. Так же как и в предыдущей теореме,

$$
\delta(x, y)=\inf _{(v, s) \in D\left(f_{x y}\right)} f_{x y}(v, s), \quad D\left(f_{x y}\right) \subseteq c_{s}(x) \times c_{s}(y) .
$$

Для $x, y \in C_{s}(U)$

$$
D\left(f_{x y}\right)=\{x\} \times\{y\}, \quad f_{x y}(v, s)=f_{y x}(s, v)=h(v, s) .
$$


Если $x \in C_{s}^{+}(U), y \in C_{s}(U)$, то

$$
\begin{aligned}
D\left(f_{x y}\right) & =c_{s}^{+}(x) \times c_{s}^{+}(y), \quad D\left(f_{y x}\right)=\{y\} \times c_{s}^{+}(x), \\
f_{x y}(v, s) & =f_{y x}(s, v)=h(v, s)+H\left(x \backslash v,\left\{u_{\infty}\right\}\right) .
\end{aligned}
$$

Случай $x \in C_{s}^{-}(U), y \in C_{s}(U)$ полностью аналогичен предыдущему; $\delta(x, y)$ и $\delta(y, x)$ получаются из определенных вьше заменой всех символов “+” на символ “-”.

Если $x \in C_{s}^{ \pm}(U), y \in C_{s}(U)$, то

$$
\begin{aligned}
D\left(f_{x y}\right) & =c_{s}(x) \times c_{s}(y), \quad D\left(f_{y x}\right)=\{y\} \times c_{s}(x) \\
f_{x y}(v, s) & =f_{y x}(s, v)=h(v, s)+H\left(x \backslash v,\left\{u_{\infty}\right\}\right)
\end{aligned}
$$

В случае, когда $x \in C_{s}^{+}(U), y \in C_{s}^{-}(U)$,

$$
\begin{gathered}
D\left(f_{x y}\right)=c_{s}^{+}(x) \times c_{s}^{-}(y), \quad D\left(f_{y x}\right)=c_{s}^{-}(y) \times c_{s}^{+}(x), \\
f_{x y}(v, s)=f_{y x}(s, v)=h(v, s)+H\left(x \backslash v,\left\{u_{\infty}\right\}\right)+H\left(y \backslash s,\left\{u_{\infty}\right\}\right) .
\end{gathered}
$$

Пусть $x \in C_{s}^{ \pm}(U), y \in C_{s}^{+}(U)$. Тогда

$$
\begin{aligned}
& D\left(f_{x y}\right)=c_{s}(x) \times c_{s}(y), \quad f_{x y}(v, s)=h(v, s)+H\left(x \backslash v,\left\{u_{\infty}\right\}\right), \\
& D\left(f_{y x}\right)=c_{s}^{+}(y) \times c_{s}(x), \quad f_{y x}(s, v)=h(s, v)+H\left(y \backslash s,\left\{u_{\infty}\right\}\right)+H\left(x \backslash v,\left\{u_{\infty}\right\}\right) .
\end{aligned}
$$

В случае $x \in C_{s}^{ \pm}(U), y \in C_{s}^{-}(U)$ величина $\delta(x, y)$ получается из вьшеуказанной заменой символов "+" на "-".

Для $x, y \in C_{s}^{ \pm}(U)$

$$
D\left(f_{x y}\right)=c_{s}(x) \times c_{s}(y), \quad f_{x y}(v, s)=h(v, s)+H\left(x \backslash v,\left\{u_{\infty}\right\}\right)+H\left(y \backslash s,\left\{u_{\infty}\right\}\right) .
$$

Соответствие $\delta$ топологии пространства $F(U)$ следует из тех же соображений, которые приводились для $o$-метрики, сконструированной при доказательстве теоремы 1. Разница лишь в том, что здесь существенную роль играет тот факт, что для фиксированного $x \in F(U) \backslash C_{s}(U)$ и переменного $v \in c_{s}(x) \quad v \rightarrow x$ влечет за собой $H\left(x \backslash v,\left\{u_{\infty}\right\}\right) \rightarrow 0$ и наоборот.

Докажем, что $o$-метрика $\delta$ удовлетворяет условиям леммы 2. Пусть $x, y \in F(U)$, $\left\{z_{1}, \ldots, z_{n}\right\} \subseteq F(U), z_{1}=x, z_{n}=y, n>2$. Для каждой пары соседних элементов $z_{i}, z_{i+1}, 1 \leqslant i \leqslant n-1$, выберем пару компактов $\left(v_{i(i+1)}, v_{(i+1) i}\right) \in D\left(f_{z_{i} z_{i+1}}\right)$, удовлетворяющую неравенству (3.1). Построим по индукции от больших номеров к меньшим компакты $w_{i(i+1)}, w_{i(i-1)} \in c_{s}\left(z_{i}\right)$ для $1<i<n, w_{12} \in c_{s}\left(z_{1}\right), w_{n(n-1)} \in c_{s}\left(z_{n}\right)$. Имеем

$$
w_{n(n-1)}=v_{n(n-1)}
$$

Пусть построены компакты $w_{n(n-1)}, w_{(n-1) n}, \ldots, w_{(i+1) i}$, где $w_{(i+1) i} \subseteq v_{(i+1) i}$. Поскольку из $(3.1) h\left(v_{i(i+1)}, v_{(i+1) i}\right)<\frac{3}{2} \delta\left(z_{i}, z_{i+1}\right)$, по определению величины $h$ существует такое $w_{i(i+1)} \subseteq v_{i(i+1)}$, что $h\left(w_{i(i+1)}, w_{(i+1) i}\right)<\frac{3}{2} \delta\left(z_{i}, z_{i+1}\right)$. Допустим, что $i>1$. Так как

$$
\operatorname{diam}\left(z_{i} \backslash v_{i(i-1)}\right)<2 H\left(z_{i} \backslash v_{i(i-1)},\left\{u_{\infty}\right\}\right)<3 \delta\left(z_{i-1}, z_{i}\right)
$$




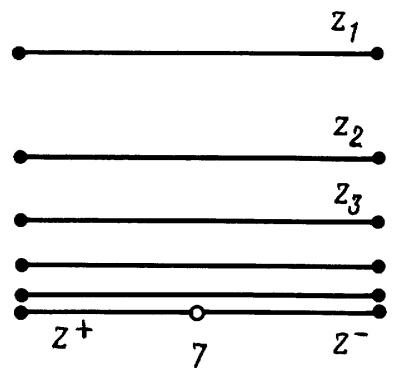

Рис. 1

(последнее неравенство следует из (3.1)), очевидно найдется такой компакт $w_{i(i-1)} \subseteq$ $v_{i(i-1)}$, что $h\left(w_{i(i-1)}, w_{i(i+1)}\right)<3 \delta\left(z_{i-1}, z_{i}\right)$.

Используя полученные неравенства, имеем

$$
\begin{aligned}
\delta\left(z_{1}, z_{n}\right) \leqslant & h\left(w_{12}, w_{n(n-1)}\right)+H\left(z_{1} \backslash w_{12},\left\{u_{\infty}\right\}\right)+H\left(z_{n} \backslash w_{n(n-1)},\left\{u_{\infty}\right\}\right) \\
\leqslant & h\left(w_{12}, w_{21}\right)+\sum_{i=2}^{n-1}\left\{h\left(w_{i(i-1)}, w_{i(i+1)}\right)+h\left(w_{i(i+1)}, w_{(i+1) i}\right)\right\} \\
& +\frac{3}{2} \delta\left(z_{1}, z_{2}\right)+\frac{3}{2} \delta\left(z_{n-1}, z_{n}\right)<7 \frac{1}{2} \sum_{i=1}^{n-1} \delta\left(z_{i}, z_{i+1}\right) .
\end{aligned}
$$

Мы доказали, что для любых $x, y \in F(U)$, где $x$ - фиксирован, из того, что величина, определенная в лемме $2, r(x, y) \rightarrow 0$ следует, что $\delta(x, y) \rightarrow 0$. Обратное очевидно. Поэтому $r(x, y)$ является $o$-метрикой, и по лемме 2 пространство $F(U) \Delta$-метризуемо,а $r(x, y)$ является соответствующей $\Delta$-метрикой. Теорема доказана.

СлЕДСТВИЕ. $F(U)$ является $T_{1}$-пространством.

Однако хаусдорфовьм пространство $F(U)$ в общем случае может и не быть.

ПримеР 1 (рис. 1). Пусть $M=\mathbb{R}, U=\mathbb{R} \times \mathbb{R} \backslash\{(0,0)\}$,

$$
\begin{array}{lll}
z^{+} \in C_{s}^{+}(U), & \pi\left(z^{+}\right)=[-1,0), & z^{+}(t) \equiv 0, \\
z^{-} \in C_{s}^{-}(U), & \pi\left(z^{-}\right)=(0,1], & z^{-}(t) \equiv 0 .
\end{array}
$$

Для каждого $i, 1 \leqslant i<\omega$,

$$
z_{i} \in C_{s}(U), \quad \pi\left(z_{i}\right)=[-1,1], \quad z_{i}(t) \equiv \frac{1}{i}
$$

Последовательность $z_{i}$, очевидно, сходится как к $z^{+}$, так и к $z^{-}$. Следовательно, $F(U)$ - не хаусдорфово.

При некоторых предположениях относительно подмножеств пространства $F(U)$ последние обладают более сильными свойствами, чем само $F(U)$. 
ТеОрема 3. Подмножсество $X$ пространства $F(U)$ метризуемо тогда и только тогда, когда оно регулярно.

ДокАЗАТЕЛЬСТво. Как мы уже выяснили, $F(U)$ имеет счетную базу. Согласно хорошо известным утверждениям регулярное пространство со счетной базой нормально и метризуемо.

$\mathrm{B}[1]$ и [2] рассмотрены свойства подмножеств $Z$ пространства $C_{s}(U)$, соответствующие простейшим свойствам множеств решений обыкновенных дифференциальных уравнений. Перечислим некоторые из них:

1) если $z \in Z$ и отрезок $I \subseteq \pi(z)$, то $\left.z\right|_{I} \in Z$;

2) если $z_{1}, z_{2} \in Z, z_{1}$ и $z_{2}$ совпадают на $\pi\left(z_{1}\right) \cap \pi\left(z_{2}\right)$, то

$$
z(t)= \begin{cases}z_{1}(t) & \text { при } t \in \pi\left(z_{1}\right), \\ z_{2}(t) & \text { при } t \in \pi\left(z_{2}\right)\end{cases}
$$

также принадлежит $Z$;

3) (аксиома компактности) для любого компакта $K \subset U$ подмножество $\{z \in Z: z \subseteq$ $K\}$ компактно.

Через $R^{i}(U)$ обозначается семейство всех пространств $Z \subseteq C_{s}(U)$, удовлетворяющих условию 1$)$.

Символ $R(U)$ обозначает множество всех пространств $Z \subseteq C_{s}(U)$, которые удовлетворяют условиям 1) и 2).

Если кроме условия 1) (условий 1 ) и 2)) вьполняется условие 3 ), то семейство таких пространств обозначается через $R_{c}^{i}(U)\left(R_{c}(U)\right)$.

Соответствующее множеству $Z \subseteq C_{s}(U)$ пространство $F_{Z}(U)$ определим как множество всех элементов пространства $F(U)$, графики которых имеют вид $[\cup \mathcal{A}]_{U}$, где $\mathcal{A}$ - некоторое упорядоченное по включению подмножество множества графиков элементов $Z$, т.е. такое подмножество, что для любых $z_{1}, z_{2} \in \mathcal{A}$ либо $z_{1} \subseteq z_{2}$, либо $z_{2} \subseteq z_{1}$.

ЗАмЕчаниЕ. Вполне очевидно, что для $Z \in R_{c}^{i}(U) F_{Z}(U) \cap C_{s}(U)=\cup\left\{c_{s}(z): z \in\right.$ $\left.F_{Z}(U)\right\}=Z$.

УТВЕРЖДЕНИЕ 3. Если $Z \in R_{c}^{i}(U)$, mo $F_{Z}(U)$ замкнуто в $F(U)$.

ДоказАТЕльство. Так как пространство $F(U)$ имеет счетную базу, достаточно доказать, что предел любой сходящейся в $F(U)$ последовательности элементов из $F_{Z}(U)$ принадлежит $F_{Z}(U)$.

Пусть $\left\{z_{k}: k<\omega\right\} \subseteq F_{Z}(U)$ и $z_{k} \rightarrow z$. Непосредственно из определения сходимости в $F(U)$ следует, что $z=\bigcup_{j<\omega} y_{j}$, где $y_{j} \in c_{s}(z), y_{j^{\prime}} \subseteq y_{j^{\prime \prime}}$ для $j^{\prime}<j^{\prime \prime}$, и к каждому $y_{j}, j<\omega$, сходится последовательность $\left\{x_{k}^{j} \in c_{s}\left(z_{k}\right): k<\omega\right\}$. Так как $Z \in R_{c}^{i}(U)$, то каждое $x_{k}^{j} \in Z$, а так как, кроме того, $U$ локально-компактно, то для каждого $j y_{j} \in Z$. Следовательно, по определению пространства $F_{Z}(U) z \in F_{Z}(U)$.

Лемма 5. Если $Z \in R_{c}^{i}(U)$, то $F_{Z}(U)$ является локально бикомпактным пространством в том смысле, что в $F_{Z}(U)$ существует база открытых мнохсеств, замыкание в $F_{Z}(U)$ каждого әлемента которой является бикомпактным (но не обязательно хаусдорфовым) пространством. 
ДокАЗАТЕЛьСТво. Поскольку $F_{Z}(U)$ обладает счетной базой и замкнуто в $F(U)$, как было доказано ранее, то достаточно доказать, что существует такая база открытых множеств в $F(U)$, что из любой последовательности точек из $F_{Z}(U)$, содержащейся в каком-либо элементе этой базы, можно выбрать подпоследовательность, сходяшуюся к некоторой точке пространства $F(U)$.

Из определения топологии пространства $F(U)$ и локальной компактности пространства $U$ следует, что семейство $\left\{V^{0}, V^{+}, V^{-}, V^{ \pm}: V\right.$ - открыто, $[V]_{U}-$ компактно $\}$ является базой $F(U)$. Пусть $H$ - один из элементов $V^{0}, V^{+}, V^{-}, V^{ \pm}$этой базы, соответствующих какому-либо $V,\left\{z_{i}: i<\omega\right\}$ - некоторая последовательность, являющаяся подмножеством множества $F_{Z}(U) \cap H$. Для каждого $i$ выберем элемент $v_{i} \in c_{s}\left(z_{i}\right) \cap$ $V$. Пусть для каждого $j, 1 \leqslant j<\omega, W_{j}$ есть $\varepsilon / j$-окрестность точки $u_{\infty}$ в $U^{*}$, где $\varepsilon=\rho\left([V], u_{\infty}\right)$.

Нетрудно проверить, что из последовательности $z_{i}$ можно выбрать подпоследовательность одного из следуюших видов, перечисленных ниже. Поэтому без ограничения общности будем считать, что последовательность $z_{i}$ относится к одному из них.

1) Существует такое $j_{0}$, что для всех $i z_{i} \subseteq U \backslash W_{j_{0}}$. Так как по определению пространства $U^{*}$ множество $U \backslash W_{j_{0}}$ компактно, $z_{i} \in C_{s}(U)$, а из замечания, сделанного выше, $z_{i} \in Z$ для всех $i$. Поскольку $Z \in R_{c}^{i}(U)$, и все $z_{i}$ принадлежат компакту, из последовательности $z_{i}$ можно выделить подпоследовательность, сходящуюся к некотоpому $z \in Z$.

2) Существует такое $j_{0}$, что для всех $\left.\left.i z_{i}\right|_{(\inf } \pi\left(z_{i}\right), \sup \pi\left(v_{i}\right)\right] \subseteq U \backslash W_{j_{0}}$, но для каждого $i z_{i} \cap W_{i} \neq \varnothing$. Очевидно, что тогда $\left\{z_{i}: i<\omega\right\} \subseteq C_{s}^{+}(U)$. При любом $i$ для каждого $j \leqslant i$ выберем элемент $y_{i}^{j} \in c_{s}^{+}\left(z_{i}\right)$ такой, что $y_{i}^{j} \cap W_{j} \neq \varnothing$ и $y_{i}^{j} \subseteq U \backslash W_{j+1}$. Так как $Z \in R_{c}^{i}(U)$, из замечания к определению пространства $F_{Z}(U) y_{i}^{j} \in Z$. Пользуясь тем, что $Z \in R_{c}^{i}(U)$, а также компактностью множеств $U \backslash W_{i}$, построим по индукции для каждого $j, 1 \leqslant j<\omega$, подпоследовательность $\left\{y_{i(j, k)}^{j}: k<\omega\right\}$ последовательности $\left\{y_{i}^{j}: i<\omega\right\}$.

Выберем из последовательности $y_{i}^{1}$ сходящуюся подпоследовательность $y_{i(1, k)}^{1}$.

Пусть построена последовательность $y_{i(j, k)}^{j}$. Последовательность $y_{i(j+1, k)}^{j+1}$ есть сходящаяся подпоследовательность последовательности $y_{i(j, k)}^{j+1}, i(j, k) \geqslant j+1$.

Для каждого $j$ последовательность $y_{i(j, k)}^{j}$ сходится к некоторому элементу $x_{j} \in Z$. При этом $\rho\left(x_{j}\left(\sup \pi\left(x_{j}\right)\right), u_{\infty}\right) \leqslant \varepsilon / j, \rho\left(x_{j}\left(\inf \pi\left(x_{j}\right)\right), u_{\infty}\right) \geqslant \varepsilon, x_{j} \subseteq x_{j+1}$. Поэтому диагональная последовательность $y_{i(j, j)}^{j}$, а следовательно, и последовательность $z_{i(j, j)}^{j}$, сходятся к элементу $x=\cup_{j=1}^{\infty} x_{j} \in C_{s}^{+}(U)$.

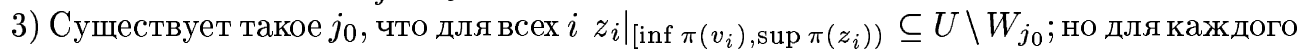
$i z_{i} \cap W_{i} \neq \varnothing$. В этом случае, полностью аналогичном предыдущему, $\left\{z_{i}: i<\omega\right\} \subseteq$ $C_{s}^{-}(U)$ и из последовательности $z_{i}$ можно выбрать подпоследовательность, сходящуюся к элементу из $C_{s}^{-}(U)$.

4) Последовательность $z_{i}^{+}=\left.z_{i}\right|_{\left.\text {[inf } \pi\left(v_{i}\right), \sup \pi\left(z_{i}\right)\right)}$ обладает свойствами последовательности $z_{i}$ в случае 2$)$, а последовательность $\left.z_{i}^{-}=\left.z_{i}\right|_{(\inf } \pi\left(z_{i}\right), \sup \pi\left(v_{i}\right)\right]$ обладает свойствами последовательности $z_{i}$ в случае 3$)$. Выделим из последовательности $z_{i}^{+}$подпоследовательность $z_{i(k)}^{+}$, сходящуюся к элементу $x^{+} \in C_{s}^{+}(U)$, а затем из последовательности $z_{i(k)}^{-}$выделим подпоследовательность $z_{i(k(l))}^{-}$, сходящуюся к элементу $x^{-} \in$ $C_{s}^{-}(U)$. Очевидно, что последовательность $z_{i(k(l))}$ сходится к элементу $x=x^{+} \cup x^{-} \in$ 
$C_{s}^{ \pm}(U)$.

Лемма доказана.

СлЕдСТВИЕ. Если $Z \in R_{c}^{i}(U)$ и пространство $F_{Z}(U)$ хаусдорфово, то $F_{Z}(U)$ локально компактно.

Теорема 4. Для $Z \in R_{c}^{i}(U)$ метризуемость $F_{Z}(U)$ равносильна хаусдорфовосmu.

ДоКАЗАТЕЛЬСТво. Здесь мы опять предъявим метрику в явном виде. Для того чтобы установить истинность утверждения, достаточно доказать, что если $F_{Z}(U)$ не метризуемо, то оно не является хаусдорфовым. Согласно утверждению $3 F_{Z}(U)$ замкнуто в $F(U)$, поэтому из леммы 4 следует, что $F_{Z}(U) o$-метризуемо любой $o$-метрикой, которой $o$-метризуемо $F(U)$. Учитьвая это, воспользуемся леммой 3 , которая позволяет свести доказательство к доказательству следующего факта.

Пусть $y \in F_{Z}(U),\left\{y_{i}: i<\omega\right\} \subseteq F_{Z}(U)$, и $\delta\left(y, y_{i}\right) \rightarrow 0$ при $i \rightarrow \infty$, где $\delta$ есть $o$-метрика, построенная при доказательстве теоремы 2, но $\lim _{i \rightarrow \infty} \delta\left(y_{i}, y\right) \neq 0$. Тогда существует $x \in F_{Z}(U), x \neq y$, к которому сходится некоторая подпоследовательность последовательности $y_{i}$, что противоречит хаусдорфовости.

Из определения величины $\delta$ следует, что при вышеуказанных условиях найдется $y^{\prime} \subseteq y, y^{\prime} \in C_{s}^{+}(U) \cup C_{s}^{-}(U)$, и последовательность $y_{i}^{\prime} \subseteq y_{i}, y_{i}^{\prime} \in C_{s}(U)$, такая, что $\delta\left(y^{\prime}, y_{i}^{\prime}\right) \rightarrow 0, \lim _{i \rightarrow \infty} \delta\left(y_{i}^{\prime}, y^{\prime}\right) \neq 0$. Поэтому без ограничения общности можно считать, что $y \in C_{s}^{+}(U)$ и $y_{i} \in C_{s}(U)$.

Для каждого $i$ существуют $s_{i} \in c_{s}^{+}\left(y_{i}\right), \quad w_{i} \in c_{s}^{+}(y)$ такие, что $h\left(w_{i}, s_{i}\right)<$ $2 \delta\left(y, y_{i}\right)$ и $H\left(y \backslash w_{i},\left\{u_{\infty}\right\}\right)<2 \delta\left(y, y_{i}\right)$. Отсюда $\rho\left(y_{i}\left(\sup \pi\left(s_{i}\right)\right), u_{\infty}\right) \rightarrow 0$ при $i \rightarrow$ $\infty$. Поэтому для каждого $\varepsilon>0$ существует подпоследовательность $y_{i(j)}$ такая, что $\rho\left(y_{i(j)}\left(\sup \pi\left(s_{i(j)}\right)\right), u_{\infty}\right)<\varepsilon / j$. Поскольку $\lim _{i \rightarrow \infty} \delta\left(y_{i}, y\right) \neq 0$, а следовательно, $\lim _{i \rightarrow \infty} H\left(y_{i} \backslash s_{i},\left\{u_{\infty}\right\}\right) \neq 0$, для некоторого $\varepsilon>0$ сушествует подпоследовательность $y_{i(k)}$ такая, что $H\left(y_{i(k)},\left\{u_{\infty}\right\}\right)>\varepsilon$ для всех $k$. Без ограничения общности будем считать, что $i=i(j)=i(k)$. Исходя из двух последних фактов, для каждого $i$ выберем $t_{i} \in \pi\left(y_{i}\right), t_{i}>\sup \pi\left(s_{i}\right)$, такое, что $\rho\left(y_{i}\left(t_{i}\right), u_{\infty}\right)>\varepsilon$. Положим $z_{i}=\left.y_{i}\right|_{\left[\sup \pi\left(s_{i}\right), t_{i}\right]}$.

Мы оказались в ситуации, описанной в случае 3 ) при доказательстве леммы 5 . Согласно соответствующим результатам из последовательности $z_{i}$ можно выбрать подпоследовательность $z_{i(k)}$, сходящуюся к некоторому $x \in F_{Z}(U) \cap C_{s}^{-}(U), x \neq y \in F_{Z}(U) \cap$ $C_{s}^{+}(U)$. Соответствующая последовательность $y_{i(k)}$ тоже сходится к $x$.

Таким образом, пространство $F_{Z}(U)$ метризуемо метрикой $r^{\prime}(x, y)=\max \{r(x, y)$; $r(y, x)\}$, где $r(x, y)-\Delta$-метрика, построенная при доказательстве теоремы 2 . Теорема доказана.

Однако, если пространство $Z$ не удовлетворяет аксиоме компактности, теорема 4 теряет свою силу даже для класса $R(U)$ независимо от замкнутости $F_{Z}(U)$ в $F(U)$, о чем свидетельствует следуюший

ПРимеР 2 (рис. 2). Положим $M=\mathbb{R}$,

$$
\begin{aligned}
& U=\mathbb{R} \times \mathbb{R} \backslash(\{(1,0)\} \cup\{(2 k, 0): 1 \leqslant k<\omega\}), \\
& z_{0} \in C_{s}^{+}(U), \quad \pi\left(z_{0}\right)=[0,1), \quad z_{0}(t) \equiv 0 .
\end{aligned}
$$

Для каждого $i, 1 \leqslant i<\omega$,

$$
z_{i} \in C_{s}^{-}(U), \quad \pi\left(z_{i}\right)=(2 i, 2 i+1], \quad z_{i}(t) \equiv 0 .
$$



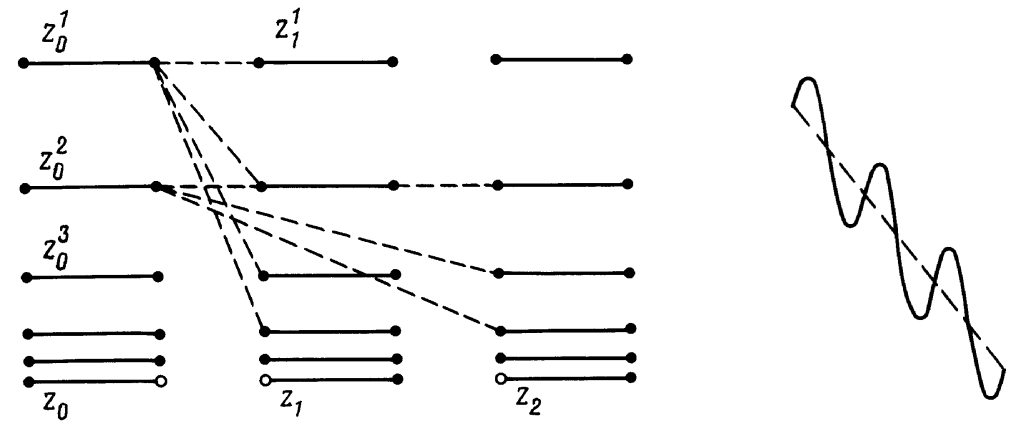

Рис. 2

Для каждого $i \geqslant 0$ определим последовательность $\left\{z_{i}^{j}: 1 \leqslant j<\omega\right\} \subset C_{s}(U)$, сходящуюся только к $z_{i}$ :

$$
\pi\left(z_{i}^{j}\right)=\left[\pi\left(z_{i}\right)\right]_{\mathbb{R}}, \quad z_{i}^{j}(t) \equiv \frac{1}{j}
$$

Для каждых $i, j \geqslant 1$ проведем отрезок, соединяющий точку $z_{0}^{i}\left(\sup \pi\left(z_{0}^{i}\right)\right)$ с точкой $z_{i}^{j}\left(\inf \pi\left(z_{i}^{j}\right)\right)$, являющийся графиком функции $w_{i j} \in C_{s}(U)$. Определим элемент $x_{i j} \in$ $C_{s}(U)$ следуюшим образом:

$$
\begin{gathered}
\pi\left(x_{i j}\right)=\left[\inf \pi\left(z_{0}\right), \sup \pi\left(z_{i}\right)\right],\left.\quad x_{i j}\right|_{\pi\left(z_{0}\right)}=z_{0}^{i},\left.\quad x_{i j}\right|_{\pi\left(z_{i}\right)}=z_{i}^{j}, \\
\left.x_{i j}\right|_{\pi\left(x_{i j}\right) \backslash\left(\pi\left(z_{0}\right) \cup \pi\left(z_{i}\right)\right)}(t)=w_{i j}(t)+\sin (2 \pi(i+j) t) .
\end{gathered}
$$

Мы получили множество

$$
Z=\bigcup_{0 \leqslant i<\omega} c_{s}\left(z_{i}\right) \cup \bigcup_{1 \leqslant i, j<\omega} c_{s}\left(x_{i j}\right) \subset R(U) .
$$

Рассмотрим соответствуюшее пространство $F_{Z}(U)$. Докажем, что оно хаусдорфово. Поскольку $F(U)$ удовлетворяет первой аксиоме счетности, достаточно доказать, что не существует последовательности, сходящейся сразу к двум точкам. Причем очевидно, что достаточно рассматривать последовательности, состоящие из элементов множества $C_{s}(U) \cap F_{Z}(U)$. Легко можно проверить, что такие пространства как $F_{c_{s}\left(x_{i j}\right)}(U)$ и $F_{c_{s}\left(z_{i}\right)}(U)$ являются хаусдорфовьми. Поэтому достаточно ограничиться последовательностями вида $\left\{y_{k} \in c_{s}\left(x_{i(k) j(k)}\right): k<\omega\right\}$.

Строение элементов $x_{i j}$ таково, что для каждого бесконечного семейства $X=\left\{x_{i j}\right.$ : $i \in A, j \in B\}$ и каждой точки $t \in C=\mathbb{R} \backslash \bigcup_{0 \leqslant i<\omega}\left\langle\pi\left(z_{i}\right)\right\rangle_{\mathbb{R}}$ множество $t \times \mathbb{R} \cap[\cup X]_{U} \backslash \cup X$ обязательно содержит всюду плотное подмножество множества $\{t\} \times I$, где $I$ - единичньй отрезок. Это делает невозможным сходимость какой-либо последовательности $y_{k} \in c_{s}\left(x_{i(k) j(k)}\right)$ к элементу из $C_{s}(C \times \mathbb{R})$. Здесь нарушается аксиома компактности. Поэтому, перебрав возможные случаи, которых немного, нетрудно убедиться в том, что любая последовательность $y_{k} \in c_{s}\left(x_{i(k) j(k)}\right)$ может сходиться только к одному элементу. Легко также проверяется, что пространства $Z$ и $F_{Z}(U)$ замкнуты в $C_{s}(U)$ и $F(U)$ соответственно. 
Пусть $F_{Z}(U)$ метризуемо метрикой $\rho$; тогда для каждых $i \geqslant 0$ и $k>0$ найдется стандартная окрестность $G_{i}^{k}=G\left(z_{i}, v_{i}^{k}, V_{i}^{k}\right)$, принадлежашая $1 / k$-окрестности точки $z_{i}$. Так как для каждого $i z_{i}^{j}$ сходится к $z_{i}$ при $j \rightarrow \infty$, для любого $k$ найдется $z_{i}^{j(i, k)} \in$ $G_{i}^{k}$. При этом каждый элемент $x_{m n} \supseteq z_{i}^{j(i, k)}$ будет тоже принадлежать окрестности $G_{i}^{k}$, а следовательно, находиться на расстоянии, меньшем $1 / k$ от $z_{i}$.

Положим $i(k)=j(0, k), j(k)=j(i(k), k)$. Соответствующий элемент $x_{i(k) j(k)}$ будет находиться на расстоянии, меньшем $1 / k$ от $z_{0}$ и от $z_{i(k)}$. Следовательно,

$$
\rho\left(z_{0}, z_{i(k)}\right) \leqslant \rho\left(z_{0}, x_{i(k) j(k)}\right)+\rho\left(x_{i(k) j(k)}, z_{i(k)}\right)<\frac{2}{k},
$$

т.е. $z_{i(k)} \rightarrow z_{0}$ при $k \rightarrow \infty$, что явно противоречит топологии пространства $F(U)$. Таким образом, пространство $F_{Z}$ хаусдорфово, но не метризуемо.

4. Пространство $F^{*}(U)$. Пространством $F^{*}(U)$ мы будем называть подпространство $\{(t, z): t \in \pi(z), z \in F(U)\}$ топологического произведения $\mathbb{R} \times F(U)$.

ЛЕмма 6. Пространство $F^{*}(U)$ метризуемо.

Для доказательства леммы определим соответствующую метрику $r^{*}$. Каждьй элемент $(t, z)$ пораждает два элемента $z_{t}^{+}=\left.z\right|_{[t,+\infty) \cap \pi(z)} \in F^{+}(U), z_{t}^{-}=\left.z\right|_{(-\infty, t] \cap \pi(z)} \in$ $F^{-}(U)$. Пусть $\left(t_{1}, z_{1}\right),\left(t_{2}, z_{2}\right) \in F^{*}(U)$. Положим

$$
r^{*}\left(\left(t_{1}, z_{1}\right),\left(t_{2}, z_{2}\right)\right)=r^{+}\left(z_{1 t_{1}}^{+}, z_{2 t_{2}}^{+}\right)+r^{-}\left(z_{1 t_{1}}^{-}, z_{2 t_{2}}^{-}\right),
$$

где $r^{+}$и $r^{-}-$метрики на множествах $F^{+}(U)$ и $F^{-}(U)$, определенные при доказательстве теоремы 1.

Вполне очевидно, что $(t, z) \rightarrow\left(t_{0}, z_{0}\right)$ тогда и только тогда, когда $z_{t}^{+} \rightarrow z_{0 t_{0}}^{+}$и $z_{t}^{-} \rightarrow$ $z_{0 t_{0}}^{-}$. Отсюда следует, что $r^{*}$ является $o$-метрикой, задающей топологию пространства $F^{*}(U)$. Аксиомы симметрии и треугольника для $r^{*}$ проверяются тривиально.

Таким образом, $F^{*}(U)$ метризуемо метрикой $r^{*}$.

СлЕДСТВИЕ. Если для пространства $Z \subseteq F(U)$ существует $t_{0}$, принадлежащее области определения каэдого $z \in Z$, то $Z$ метризуемо.

ДокАЗАтЕльство. Отображение $f: Z \rightarrow F^{*}(U), f(z)=\left(t_{0}, z\right)$, очевидно, является гомеоморфным вложением. Следовательно, $Z$ метризуемо, так же как и $F^{*}(U)$.

\section{СПИСОК ЦИТИРОВАННОЙ ЛИТЕРАТУРЫ}

[1] Федорчук В. В., Филиппов В.В.Общая топология. Основные конструкции. М.: Изд-во МГУ, 1988.

[2] Филиппов В.В.Пространства решений обыкновенных дифференциальных уравнений. М.: Изд-во МГУ, 1993.

[3] Филиппов В.В. О топологических свойствах пространств решений обыкновенных дифференциальых уравнений // Докл. РАН. 1997. Т. 352. №6. С. 735-738.

[4] Yorke J. Spaces of solutions // Lecture Notes in Operat. Res. and Math. Econom. V. 12. Berlin: Springer-Verlag, 1969. P. 383-403.

[5] Недев С.Й. о-метризуемые пространства // Труды ММО. 1971. Т. 24. С. 201-236.

Московский государственный университет

Поступило им. М.В. Ломоносова

05.07.96

Исправленньй вариант 20.05.97 